PREHOSPITAL CARE

\title{
Prehospital trauma management: a national study of paramedic activities
}

\author{
S Sukumaran, J M Henry, D Beard, R Lawrenson, M W G Gordon, J J O'Donnell, \\ A J Gray
}

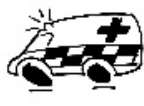

Emerg Med J 2005;22:60-63. doi: 10.1136/emj.2004.016873 See end of article for
authors' affiliations

Correspondence to: Dr S Sukumaran, Emergency Department, Royal Infirmary of Edinburgh, Old Dalkeith Road, Little France, Edinburgh EH16 4SU, UK; s_sukumaran@hotmail. com

Accepted for publication 1 September 2004

\begin{abstract}
Objectives: The benefits of prehospital trauma management remain controversial. This study aimed to compare the processes of care and outcomes of trauma patients treated by paramedics, who are trained in advanced prehospital trauma care, with those treated by ambulance technicians.

Methods: A six year prospective study was conducted of adult trauma patients attended to by the Scottish Ambulance Service and subsequently admitted to hospital. Prehospital times, interventions, triage, and outcomes were compared between patients treated by paramedics and those treated by technicians. Results: Paramedics attended more severely injured patients $(16.5 \%$ versus $13.9 \%, \mathrm{p}<0.001)$; they attended a higher proportion of patients with penetrating trauma $(6.6 \%$ versus $5.7 \%, p=0.014)$ and had longer prehospital times. Patients managed by paramedics were more likely to be taken to the intensive care unit, operating theatre or mortuary, $(11.2 \%$ versus $7.8 \%, \mathrm{p}<0.001)$ and had higher crude mortality rates $(5.3 \%$ versus $4.5 \%, p=0.07)$. However, no difference in mortality between the two groups was noted when corrected for age, Glasgow coma score and injury severity score.

Conclusions: This large scale national study shows that paramedics show good triage skills and clinical judgement when managing trauma patients. However, the value of the individual interventions they perform could not be ascertained. Further controlled trials are necessary to determine the true benefits of advanced prehospital trauma life support.
\end{abstract}

$\mathrm{T}$ rauma is the leading cause of death in adults less that 45 years of age in the United Kingdom. ${ }^{12}$ The trimodal distribution of trauma deaths described by Trunkey also highlighted a high proportion of deaths within the first hours of the trauma, which might have been prevented by earlier medical intervention. ${ }^{3}$ This prompted the proposal by the Department of Health to have at least one paramedic who was trained in advanced prehospital trauma care in all emergency ambulances in England and Wales. ${ }^{5}$ While both paramedics and ambulance technicians assess, triage, and treat trauma patients, paramedics receive a minimum of eight weeks' additional specialised training in endotracheal intubation, intravenous cannulation, fluid administration, and the use of a number of drugs. ${ }^{6}$ Despite its adoption, however, the benefits of this additional training have been questioned, as such interventions could delay the administration of definitive care to the patient. $^{7-9}$

This study aims to compare the relative contributions made by paramedics with advanced prehospital trauma care skills, and ambulance technicians, with the processes of care and outcomes of patients after trauma in Scotland.

\section{METHODS}

We analysed all prospectively identified trauma patients, aged over 13 years, (the age used in Scotland to separate paediatric from adult patients) taken by the Scottish Ambulance Service to 26 emergency departments (EDs) in Scotland from l July 1996 to 30 June 2002. Data were collected by the Scottish Trauma Audit Group (STAG), whose inclusion and exclusion criteria are listed in the box. These criteria concur with those used by the Trauma Audit and Research Network for England and Wales, UK TARN; in addition, patients who arrived in the ED in cardiopulmonary arrest whose period of resuscitation was less than 15
Inclusion and exclusion criteria of the Scottish Trauma Audit Group

Inclusion criteria:

- Patients who are admitted to hospital for at least three days, or who die as a result of trauma.

Exclusion criteria:

- Patients aged over 65 with an isolated fracture of the neck of femur or pubic ramus.

- Children aged under 13.

minutes, inter-hospital transfers, and patients managed by a doctor at scene were excluded.

Patients are audited using TRISS methodology. ${ }^{10}$ The following data were used from the STAG database: mechanism and type of injury, prehospital times (response, on-scene, transport, and total times), interventions on-scene, triage category, revised trauma scores in the ED, injury severity scores, destination from the ED, and outcome. The on-scene interventions and times were obtained from the ambulance service patient report forms (PRF), which include the type of ambulance personnel attending the patient. The personnel response to an incident (paramedic or ambulance technician) was decided by central ambulance control, but was most commonly determined by which ambulance was closest to the scene.

Data were analysed using SPSS for Windows V1l. Categorical variables were analysed using $\chi^{2}$ tests. The

Abbreviations: ISS, injury severity score; GCS, Glasgow coma score; ED, emergency department; STAG, Scottish Trauma Audit Group; SMR, standardised mortality ratios; PRF, patient report form 
Table 1 Demographic data and injury mechanism

\begin{tabular}{lllr}
\hline & Paramedic & Technician & p Value \\
\hline Total number of patients (\%) & $12339(58)$ & $9078(42)$ & \\
Median age (y) & 48 & 51 & $<0.001$ \\
Male sex (\%) & $7461(60)$ & $5183(57)$ & $<0.001$ \\
Penetrating trauma (\%) & $810(6.6)$ & $521(5.7)$ & 0.013 \\
RTA, assault or fall $>2 \mathrm{~m}$ & $5984(48.5)$ & $3780(41.6)$ & $<0.001$ \\
\hline
\end{tabular}

continuous prehospital times were of non-parametric distribution and were compared using Mann-Whitney U tests. Standardised mortality ratios (SMR) were standardised for age, Glasgow coma score (GCS), and injury severity score (ISS): eight age groups, five GCS categories, and five ISS categories.

\section{RESULTS}

Altogether 26523 patients who fulfilled STAG entry criteria were identified in the study period. Of these, 5106 (19\%) were excluded because either the PRF or the grade of ambulance response was unavailable. This left 21417 patients for analysis.

\section{Demographics and injury patterns}

Paramedics attended to $12339(58 \%)$ of patients, while ambulance technicians attended to 9078 (42\%). Most patients, $20086(93.8 \%)$ had blunt trauma. Paramedics attended more patients who had been involved in a road traffic accident, assault, and fall from over two metres (table 1).

\section{Prehospital times}

Table 2 shows ambulance response times, on-scene times, and transfer to ED times. Response times were similar in both groups, but paramedics spent longer at scene and had longer total prehospital times than technicians.

\section{Interventions}

An advanced trauma intervention, such as endotracheal intubation or cannulation, was performed on 4011 (32.5\%) of the paramedic group: 128 patients $(1.0 \%)$ were intubated at scene, and 3958 (32\%) were cannulated. Patients who received an intervention spent longer on-scene than those who did not (table 3). Patients who did not require an intervention had similar median on-scene times in both the paramedic and technician groups (14 versus 15 minutes, $\mathrm{p}=0.75$ ).

\section{Triage}

Paramedics attended proportionately more patients with an abnormal RTS (1796 (14.6\%) versus 1063 (11.7\%); p<0.001) or an ISS greater than 15 (2039 (16.5\%) versus 1,262 (13.9\%); $\mathrm{p}<0.001)$. More patients from the paramedic group were also triaged directly to the resuscitation room, regardless of RTS or ISS value (table 4). Paramedics made a "standby" call to the ED more frequently than technicians did for patients with ISS more than 15 (1186 (58.2\%) versus 578 (45.8\%); $\mathrm{p}<0.001)$. Almost all patients deemed physiologically compromised by the attending paramedic, and for whom a standby call was made, were triaged directly to the resuscitation room (98.4\%). Of the 407 physiologically compromised patients not triaged to the resuscitation room, $117(29 \%)$ were subsequently re-triaged there.

\section{Outcome measures}

Patients who had been managed by a paramedic were more likely to be taken to a "critical" destination (ICU, theatre, or mortuary) on leaving the ED than those treated by ambulance technicians (1379 (11.2\%) versus 706 (7.8\%); $\mathrm{p}<0.001)$. Paramedics attended a significantly higher proportion of patients who subsequently stayed in hospital for over four weeks ( $1284(10.4 \%)$ versus $832(9.2 \%) ; p=0.003)$. However, among those patients admitted to ICU there was no difference in the length of time spent in ICU between the two groups (median stay two days for both groups, $\mathrm{p}=0.09$ ).

\begin{tabular}{|lllll|}
\hline Table 2 & Prehospital & & & \\
\hline & Times $(\mathbf{m i n})$ & Paramedic & Technician & $\mathrm{p}$ Value \\
\hline Response & Median & 9 & 9 & 0.48 \\
& Interquartile range & $6-13$ & $6-13$ & \\
On-scene & Max & 297 & 230 & $<0.001$ \\
& Median & 17 & 15 & \\
Travel & Interquartile range & $11-25$ & $10-20$ & \\
& Max & 144 & 103 & 0.009 \\
Total time to hospital & 18 & 18 & \\
& Median & $11-27$ & $11-26$ & $<0.001$ \\
& Interquartile range & 195 & 185 & \\
& Max & 47 & 44 & \\
& Median & $36-64$ & $33-58$ & \\
& Interquartile range & 327 & 305 & \\
\hline
\end{tabular}

Table 3 On-scene times for patients receiving advanced prehospital trauma interventions in the paramedic group

\begin{tabular}{lllll}
\hline & Time (min) & Intervention & No intervention & p Value \\
\hline Intubation & Median & 20 & 17 & 0.018 \\
& Interquartile range & $15-25$ & $11-25$ & $<0.001$ \\
Cannulation, & Median & 23 & 14 & \\
no fluids & Interquartile range & $18-33$ & $10-20$ & $<0.001$ \\
Cannulation & Median & 25 & 14 & \\
with fluids & Interquartile range & $18-35$ & $10-20$ & \\
\hline
\end{tabular}


Table 4 Number and percentage of patients triaged directly to the resuscitation room

\begin{tabular}{llll}
\hline $\begin{array}{l}\text { Triage to } \\
\text { resuscitation room }\end{array}$ & Paramedic & Technician & p Value \\
\hline All patients & $5750 / 12339$ & $3306 / 9078$ & $<0.001$ \\
& $46.6 \%$ & $36.4 \%$ & \\
ISS $\leqslant 15$ & $4017 / 10300$ & $2352 / 7816$ & $<0.001$ \\
& $39.0 \%$ & $30.1 \%$ & \\
ISS $>15$ & $1733 / 2039$ & $954 / 1262$ & $<0.001$ \\
& $85.0 \%$ & $75.6 \%$ & \\
RTS $<7.84$ & $1567 / 1796$ & $840 / 1063$ & $<0.001$ \\
& $87.2 \%$ & $79.0 \%$ & \\
RTS normal & $4183 / 10543$ & $2466 / 8015$ & $<0.001$ \\
& $39.7 \%$ & $30.8 \%$ & \\
\hline
\end{tabular}

A total of 1063 patients (5\%) died. There was no difference in mortality rates between the paramedic treated and technician treated patients when age, GCS on arrival in the ED, and ISS were controlled for (SMR in the paramedic group $0.9984,95 \%$ CI 0.9219 to 1.0749 , and 1.0025 in the technician group, $95 \%$ CI 0.9053 to $1.0998 ; p=1.00$ ). There was also no significant difference between the two groups when patients with penetrating injuries were compared (64 versus 52 patients; $\mathrm{p}=0.20$ ). However, the paramedic treated blunt trauma patients had a higher mortality rate (591 $(5.1 \%)$ versus $356(4.2 \%) ; p=0.001)$. Their mortality rate was higher in the ED, (163 (1.3\%) versus $80(0.9 \%)$; $p=0.003)$, but not within one day of admission (335 (2.7\%) versus 209 $(2.3 \%) ; \mathrm{p}=0.06)$.

\section{DISCUSSION}

This is the largest study to date, conducted in the UK, comparing paramedic activities with those of ambulance technicians in the management of the trauma patient. The large sample size and high quality data showed small but significant differences in the groups studied. We found that patients treated by a paramedic were younger, and more likely to have suffered a penetrating injury. Paramedic treated patients also tended to have more serious injuries and a greater degree of physiological compromise. A third of their patients were cannulated, intubated, or both, and these interventions were associated with longer on-scene times. Prehospital times were consistent with other studies from the UK. ${ }^{11-13}$

This is the first national study to show paramedics' ability to correctly identify patients who required triage to a resuscitation area. Triaging increased numbers of patients to the resuscitation room and this could have significant resource implications if practised indiscriminately. However, as a higher proportion of paramedic treated patients went to a critical destination from the ED, it suggests good decision making and appropriate triage skills. The informal targeting of paramedics to the severely injured that occurs in practice is likely to be in recognition of these capabilities.

There is however continued uncertainty about the benefits of the prehospital interventions they perform on trauma patients. In this study, there was no decrease in mortality for paramedic treated patients after correction for age, GCS on arrival in the ED, and ISS was made. Admittedly the two groups under comparison were not strictly matched, and we could not take into account the regional variations in prehospital service provision across the country. Nevertheless, these findings are similar to those in an earlier paper from Edinburgh, which noted that while paramedics delivered an improved process of care, their activities did not significantly reduce mortality or length of stay in intensive care. ${ }^{11}$ A health technology assessment (HTA) paper from 1998 came to a similar conclusion, although interestingly it found decreased morbidity at six months for survivors who had received prehospital trauma life support. ${ }^{14}$

The benefits of prehospital fluid therapy in particular have been frequently questioned. ${ }^{15-19}$ A recent trial by the HTA found no improvement in mortality rates or composite outcomes in trauma patients randomised to a conventional prehospital fluid replacement regimen..$^{15}$ The authors wondered if this was because the delay to definitive care attributable to performing cannulation outweighed any benefits from receiving fluids. Using computer modelling, Lewis et al showed that fluids seemed to be beneficial only where prehospital times exceed 30 minutes. ${ }^{20}$ A policy of "scoop and run" has been proposed instead by several authors, ${ }^{71721}$ with cannulation being performed "en route" to shorten prehospital times. One study of en route cannulation in a moving ambulance reported a success rate of $95 \% .^{22}$

Prehospital endotracheal intubation of the trauma patient has also come under scrutiny. In 1985 Pepe suggested that intubation provides the best method of airway control in patients with changed levels of consciousness, shock, or an unprotected airway. ${ }^{23}$ This however has since been disputed: Davis et al showed worse outcomes in patients with severe head injury who underwent rapid sequence intubation at scene. ${ }^{24}$ The benefit of this intervention to patients with other injury patterns is also unclear. ${ }^{25}{ }^{26}$ Complication and failure rates are also higher than in non-trauma patients. ${ }^{27}$ This may be attributable to skill retention being difficult, given the low incidence of trauma intubations that occur in practice. In addition, UK paramedics are not trained in drug assisted intubation, and thus may be unable to control for potentially lethal autonomic and intracranial pressure changes during the procedure. It is also an intervention that prolonged prehospital times in our study, although the three minute delay that occurred may not be clinically significant.

In conclusion, this study shows that advanced prehospital trauma care training improves identification of the seriously injured patient who will need resuscitation or intensive care. However, no reduction in mortality was noted when the interventions it teaches were practised. More research is necessary to decide if selected patients warrant a "scoop and run" policy of prehospital trauma care.

\section{ACKNOWLEDGEMENTS}

The Scottish Ambulance Service personnel. STAG local coordinators.

\section{Authors' affiliations}

S Sukumaran, A J Gray, Emergency Department, Royal Infirmary of Edinburgh, UK

J M Henry, D Beard, Scottish Trauma Audit Group

$R$ Lawrenson, Scottish Ambulance Service

M W G Gordon, Emergency Department, Southern General Hospital, Glasgow, UK

J J O'Donnell, Emergency Department, University College Hospital, Galway, Ireland

Funding: none.

Competing interests: none declared.

\section{REFERENCES}

1 National Statistics Office. Review of the registrar general on deaths by cause, sex and age in England and Wales. London: National statistics Office, 2001.

2 General Register Office. Deaths: numbers and rates by sex, age and cause, Scotland 2002. Vital events reference table. Edinburgh: General Register Office, 2002.

3 Trunkey DD. Initial treatment of patients with extensive trauma. N Engl J Med 1991;324:1259-63

4 Trunkey DD. Trauma. Sci Am 1983;249:20-7.

5 Merifield AJ. The modern ambulance service. Report by working group to NHS management executive. London: NHS, 1990.

6 IHCD. Ambulance service paramedic training manual. London: IHCD, 2001 
7 Gold CR. Pre-hospital advanced life support vs "scoop and run" in trauma management. Ann Emerg Med 1987; 16:797-801.

8 Sampalis JS, Lavoie A, Williams JI, et al. Impact of on-site care, pre-hospital time and level of in-hospital care on survival in severely injured patients. J Trauma 1993:34:252-61.

9 Liberman M, Mulder D, Sampalis J. Advanced or basic life support for trauma: meta-analysis and critical review of the literature. J Trauma 2000;49:584-99.

10 Boyd CR, Tolson MA, Copes WS. Evaluating trauma care: the TRISS method. Trauma score and the injury severity score. J Trauma 1987;27:370-8.

11 Rainer TH, Houlihan KPG, Robertson CE, et al. An evaluation of paramedic activities in prehospital trauma care. Injury 1997;28:623-7.

12 Guly UM, Mitchell RG, Cook R, et al. Paramedics and technicians are equally successful at managing cardiac arrests outside hospital. BMJ 1995:310:1091-4.

13 Goodacre S, Gray A, McGowan A. On-scene times for trauma patients in West Yorkshire. J Accid Emerg Med 1997;14:283-5.

14 Nicholl J, Hughes S, Dixon S, et al. The costs and benefits of paramedic skills in prehospital trauma care. Health Technol Assess 1998;2.

15 Turner J, Nicholl J, Webber L, et al. A randomised controlled trial of prehospital intravenous fluid replacement therapy in serious trauma. Health Technol Assess 2000;4.

16 Bickell WH, Wall MJ, Pepe PE, et al. Immediate versus delayed fluid resuscitation for hypotensive patients with penetrating torso injuries. N Engl J Med 1994;331:1105-9.

17 Smith JP, Bodai BI, Hill AS, et al. Pre-hospital stabilization of critically injured patients: a failed concept. J Trauma 1985;25:65-70.
18 Kaweski SM, Sise MJ, Virgilio RW. The effect of prehospital fluids on survival in trauma patients. J Trauma 1990;30:1215-18.

19 Sampalis JS, Tammim H, Denis R, et al. Ineffectiveness of on-site intravenous lines: is prehospital time the culprit? J Trauma 1997:43:608-15.

20 Lewis FR. Pre-hospital intravenous fluid therapy: Physiologic computer modelling. J Trauma 1986;26:804-11.

21 Blaisdell FW. Trauma myths and magic: 1984 Fitts Lecture. J Trauma 1985;25:856-63.

22 Slovis CM, Herr EW, Londorf D, et al. Success rates for initiation of intravenous therapy en route by prehospital care providers. Am J Emerg Med 1990;8:305-7.

23 Pepe PE, Copass MK, Joyce TH, et al. Prehospital endotracheal intubation: rationale for training emergency medical personnel. Ann Emerg Med $1985 ; 14: 1085-92$.

24 Davis DP, Hoyt DB, Ochs M, et al. The effect of paramedic rapid sequence intubation on outcome in patients with severe traumatic brain injury. J Trauma 2003;54:444-53.

25 Eckstein M, Chan L, Schneir A, et al. Effect of prehospital advanced life support on outcomes of major trauma patients. J Trauma 2000;48:643-8.

26 Ruchholtz S, Waydhas C, Ose C, et al. Prehospital intubation in severe thoracic trauma without respiratory insufficiency: a matched-pair analysis based on the Trauma Registry of the German Trauma Society. J Trauma 2002;52:879-86

27 Stewart RD, Paris PM, Winter PM, et al. Field endotracheal intubation by paramedic personnel: success rates and complications. Chest 1984;85:341-5. 